Article

\title{
Hyers-Ulam Stability of Lagrange's Mean Value Points in Two Variables
}

\author{
Soon-Mo Jung ${ }^{1, *(1)}$ and Ji-Hye Kim ${ }^{2}$ \\ 1 Mathematics Section, College of Science and Technology, Hongik University, Sejong 30016, Korea \\ 2 Department of Mathematics Education, Korea National University of Education, Cheongjusi, \\ Chungbuk 28173, Korea; hyekj83@naver.com \\ * Correspondence: smjung@hongik.ac.kr
}

Received: 30 September 2018; Accepted: 22 October 2018; Published: 25 October 2018

Abstract: Using a theorem of Ulam and Hyers, we will prove the Hyers-Ulam stability of two-dimensional Lagrange's mean value points $(\eta, \xi)$ which satisfy the equation, $f(u, v)-f(p, q)=$ $(u-p) f_{x}(\eta, \xi)+(v-q) f_{y}(\eta, \xi)$, where $(p, q)$ and $(u, v)$ are distinct points in the plane. Moreover, we introduce an efficient algorithm for applying our main result in practical use.

Keywords: Hyers-Ulam stability; mean value theorem; Lagrange's mean value point; two-dimensional Lagrange's mean value point

\section{Introduction}

The stability problem for functional equations or differential equations began with the well-known question of Ulam [1]:

Let $G_{1}$ and $G_{2}$ be a group and a metric group with a metric $d(\cdot, \cdot)$, respectively. Given $\varepsilon>0$, does there exist a $\delta>0$ such that if a function $h: G_{1} \rightarrow G_{2}$ satisfies the inequality $d(h(x y), h(x) h(y))<\delta$ for all $x, y \in G_{1}$, then there exists a homomorphism $H: G_{1} \rightarrow G_{2}$ with $d(h(x), H(x))<\varepsilon$ for all $x \in G_{1}$ ?

In short, the Ulam's question states as follows: Under what conditions does there exist an additive function near an approximately additive function?

In 1941, Hyers [2] gave a partial solution to the question of Ulam under the assumption that relevant functions are defined on Banach spaces.

Theorem 1 (Hyers [2]). Given $\delta>0$, assume that $f: E_{1} \rightarrow E_{2}$ is a function between Banach spaces such that

$$
\|f(x+y)-f(x)-f(y)\| \leq \delta
$$

for all $x, y \in E_{1}$. Then the limit

$$
A(x):=\lim _{n \rightarrow \infty} \frac{1}{2^{n}} f\left(2^{n} x\right)
$$

exists for each $x \in E_{1}$ and $A: E_{1} \rightarrow E_{2}$ is the unique additive function such that

$$
\|f(x)-A(x)\| \leq \delta
$$

for any $x \in E_{1}$.

Based on Theorem 1, we say that the Cauchy additive functional equation, $f(x+y)=f(x)+f(y)$, has (or satisfies) the Hyers-Ulam stability or it is stable in the sense of Hyers and Ulam. Since then, the 
stability problems for several functional equations and differential equations have been extensively investigated by many mathematicians (see [3-15] and the references therein).

Let $f: \mathbb{R}^{2} \rightarrow \mathbb{R}$ be a function with continuous partial derivatives $f_{x}$ and $f_{y}$ and let $L$ be the line segment joining the distinct points $(p, q)$ and $(u, v)$ in $\mathbb{R}^{2}$. If a point $(\eta, \xi)$ on the line segment $L$ satisfies the equation

$$
f(u, v)-f(p, q)=(u-p) f_{x}(\eta, \xi)+(v-q) f_{y}(\eta, \xi),
$$

then $(\eta, \xi)$ is called a two-dimensional Lagrange's mean value point of $f$ in $L$.

In Section 3, we prove the Hyers-Ulam stability of two-dimensional Lagrange's mean value points. The main result of this paper is an extension and a generalization of the previous work [16] (Theorem 2.2) (or see Theorem 4 below). Moreover, we introduce an efficient method for applying our main theorem (Theorem 6) in the practical use.

\section{Preliminaries}

The concept of Hyers-Ulam stability can be applied to the case of other mathematical objects. Ulam and Hyers [17] seem to be the first mathematicians who applied the concept of Hyers-Ulam stability to differential expressions.

Theorem 2 (Ulam and Hyers [17]). Assume that $f: \mathbb{R} \rightarrow \mathbb{R}$ is $n$ times differentiable in a neighborhood $N$ of a point $t_{0}$ and $f^{(n)}\left(t_{0}\right)=0$ and $f^{(n)}(t)$ changes sign at $t_{0}$. For any $\varepsilon>0$, there corresponds a $\delta>0$ such that for every function $g: \mathbb{R} \rightarrow \mathbb{R}$ which is $n$ times differentiable in $N$ and satisfies $|f(t)-g(t)|<\delta$ for all $t \in N$, there exists a point $t_{1} \in N$ such that $g^{(n)}\left(t_{1}\right)=0$ and $\left|t_{1}-t_{0}\right|<\varepsilon$.

Given an $\varepsilon>0$, if we choose a sufficiently small $\delta>0$, Theorem 2 would certainly be true but the choice of sufficiently small $\delta$ imposes a constraint on practical use of this theorem. Indeed, we are interested in choosing the $\delta$ as large as possible. Therefore, we introduce an algorithm for the choice of $\delta$ strongly based on the proof of ([17], Theorem 1 ).

Remark 1. Assume that $\varepsilon$ and $t_{0}$ be the quantities given in Theorem 2. The following steps provide us with an efficient method for choosing the $\delta$ :

(i) $\varepsilon$ is chosen less than the radius of $N$;

(ii) we choose $t_{2}, t_{3}$ and $\alpha$ such that $\left|t_{2}-t_{0}\right|<\frac{\varepsilon}{2},\left|t_{3}-t_{0}\right|<\frac{\varepsilon}{2}, 0<\alpha<\frac{\varepsilon}{2 n}$, and $\Delta_{\alpha}^{n} f\left(t_{2}\right) \Delta_{\alpha}^{n} f\left(t_{3}\right)<0$, where $\Delta_{\alpha}^{n} f(t)=\Delta_{\alpha}\left\{\Delta_{\alpha}^{n-1} f(t)\right\}$ and $\Delta_{\alpha} f(t)=f(t+\alpha)-f(t)$;

(iii) we choose the $\delta$ as large as possible with $0<\delta<\min \left\{\frac{1}{2^{n}}\left|\Delta_{\alpha}^{n} f\left(t_{2}\right)\right|, \frac{1}{2^{n}}\left|\Delta_{\alpha}^{n} f\left(t_{3}\right)\right|\right\}$.

In 1958, Flett [18] proved a variant of Lagrange's mean value theorem: If a function $f:[a, b] \rightarrow$ $\mathbb{R}$ is continuously differentiable and $f^{\prime}(a)=f^{\prime}(b)$, then there exists a point $\eta \in(a, b)$ such that $f(\eta)-f(a)=f^{\prime}(\eta)(\eta-a)$.

A similar problem as the Ulam's question can be formulated for the mean value points:

If a function $f$ has a mean value point $\eta$ and $g$ is a function quite near to $f$, does $g$ have a mean value point near $\eta$ ?

Indeed, Das, Riedel and Sahoo [19] examined the stability problem for Flett's mean value points. Unfortunately, there were some errors in the proof of the main theorem of [19]. In 2009, Lee, Xu and Ye [20] proved the Hyers-Ulam stability of the Sahoo-Riedel's points. We remind that for a differentiable function $f:[a, b] \rightarrow \mathbb{R}$, a point $\eta \in(a, b)$ is called a Sahoo-Riedel's point of $f$ in $(a, b)$ provided $\eta$ satisfies $f(\eta)-f(a)=f^{\prime}(\eta)(\eta-a)-\frac{f^{\prime}(b)-f^{\prime}(a)}{2(b-a)}(\eta-a)^{2}$. Moreover, they obtained the following theorem concerning the stability of Flett's points as a corollary. 
Theorem 3 (Lee et al. [20]). Assume that $f, h:[a, b] \rightarrow \mathbb{R}$ are differentiable functions and $\eta$ is a Sahoo-Riedel's point of $f$ in $(a, b)$. If $f$ is twice differentiable at $\eta$ and

$$
f^{\prime \prime}(\eta)(\eta-a)-2 f^{\prime}(\eta)+\frac{2(f(\eta)-f(a))}{\eta-a} \neq 0,
$$

then for any $\varepsilon>0$ and any neighborhood $N \subset(a, b)$ of $\eta$, there exists $a \delta>0$ with the property that for every $h$ satisfying $|h(x)-h(a)-(f(x)-f(a))|<\delta$ for $x \in N$ and $h^{\prime}(b)-h^{\prime}(a)=f^{\prime}(b)-f^{\prime}(a)$, there exists a Sahoo-Riedel's point $\xi \in N$ of $h$ with $|\xi-\eta|<\varepsilon$.

Thereafter, Găvrută, Jung and Li [16] have proved the stability of the Lagrange's mean value point which is a point $\eta \in(a, b)$ of a differentiable function $f:[a, b] \rightarrow \mathbb{R}$ satisfying $\frac{f(b)-f(a)}{b-a}=f^{\prime}(\eta)$.

Theorem 4 (Găvrută et al. [16]). Let $a, b, \eta$ be real numbers satisfying $a<\eta<b$. Assume that $f: \mathbb{R} \rightarrow \mathbb{R}$ is a twice continuously differentiable function and $\eta$ is the unique Lagrange's mean value point of $f$ in an open interval $(a, b)$ and moreover that $f^{\prime \prime}(\eta) \neq 0$. Suppose $g: \mathbb{R} \rightarrow \mathbb{R}$ is a differentiable function. Then, for any given $\varepsilon>0$, there exists $a \delta>0$ with the property that if $|f(x)-g(x)|<\delta$ for all $x \in[a, b]$, then there is $a$ Lagrange's mean value point $\xi \in(a, b)$ of $g$ with $|\xi-\eta|<\varepsilon$.

We can extend the intimate concept of Lagrange's mean value points to the two-dimensional cases. We will now introduce the two-dimensional Lagrange's mean value theorem (see [21] (Theorem 4.1)). Courant introduced the following theorem in his book [22] (or see Sahoo and Riedel [21] (Theorem 4.1)).

Theorem 5 (Courant [22]). For every function $f: \mathbb{R}^{2} \rightarrow \mathbb{R}$ with continuous partial derivatives $f_{x}$ and $f_{y}$ and for all distinct points $(p, q)$ and $(u, v)$ in $\mathbb{R}^{2}$, there exists an intermediate point $(\eta, \xi)$ on the line segment $L$ joining the points $(p, q)$ and $(u, v)$ such that $f(u, v)-f(p, q)=(u-p) f_{x}(\eta, \xi)+(v-q) f_{y}(\eta, \xi)$.

The geometrical interpretation of Theorem 5 is that the difference between values of the function at points $(u, v)$ and $(p, q)$ is equal to the differential at an intermediate point $(\eta, \xi)$ on the line segment joining those two points.

\section{Main Theorem}

Assume that $f: \mathbb{R}^{2} \rightarrow \mathbb{R}$ is a function with continuous partial derivatives $f_{x}$ and $f_{y}$ and $L$ is the line segment joining the distinct points $(p, q)$ and $(u, v)$ in $\mathbb{R}^{2}$. We remind that an intermediate point $(\eta, \xi)$ on $L$ is called a two-dimensional Lagrange's mean value point of $f$ in $L$ provided that the point $(\eta, \xi)$ satisfies the equation

$$
f(u, v)-f(p, q)=(u-p) f_{x}(\eta, \xi)+(v-q) f_{y}(\eta, \xi)
$$

By making use of Theorem 2, we will prove our main theorem concerning the Hyers-Ulam stability of the two-dimensional Lagrange's mean value points.

Theorem 6. Assume that $L$ is the line segment joining two distinct points $(p, q)$ and $(u, v)$ and that $f$ : $\mathbb{R}^{2} \rightarrow \mathbb{R}$ is a twice continuously partial differentiable function. Suppose $\left(\eta_{0}, \xi_{0}\right)$ is the unique two-dimensional Lagrange's mean value point of $f$ in $L$ and the point $\left(\eta_{0}, \xi_{0}\right)$ satisfies

$$
h^{2} f_{x x}\left(\eta_{0}, \xi_{0}\right)+2 h k f_{x y}\left(\eta_{0}, \xi_{0}\right)+k^{2} f_{y y}\left(\eta_{0}, \xi_{0}\right) \neq 0,
$$

where $h=u-p$ and $k=v-q$. Then, for any given $\varepsilon>0$, there corresponds $a \delta>0$ such that if $a$ partial differentiable function $g: \mathbb{R}^{2} \rightarrow \mathbb{R}$ satisfies $|f(x, y)-g(x, y)|<\delta$ for all $(x, y) \in L$, then there is a two-dimensional Lagrange's mean value point $\left(\eta_{1}, \xi_{1}\right)$ of $g$ in $L$ with $\left|\left(\eta_{0}, \xi_{0}\right)-\left(\eta_{1}, \xi_{1}\right)\right|<\varepsilon$. 
Proof. We know that the coordinates of each point on the line segment $L$ are given by $(p+h t, q+k t)$ for some $t \in[0,1]$. We define an auxiliary function $F: \mathbb{R} \rightarrow \mathbb{R}$ by $F(t):=f(p+h t, q+k t)$ and calculate its derivative as

$$
F^{\prime}(t)=h f_{x}(p+h t, q+k t)+k f_{y}(p+h t, q+k t)
$$

We define another auxiliary function $G_{f}: \mathbb{R} \rightarrow \mathbb{R}$ by

$$
G_{f}(t):=t f(u, v)+(1-t) f(p, q)-F(t)
$$

for all $t \in \mathbb{R}$. Obviously, $G_{f}$ is twice continuously differentiable and $G_{f}(0)=G_{f}(1)=0$. Hence, by the Rolle's theorem, there exists an $t_{0} \in(0,1)$ with

$$
\begin{aligned}
G_{f}^{\prime}\left(t_{0}\right) & =f(u, v)-f(p, q)-F^{\prime}\left(t_{0}\right) \\
& =f(u, v)-f(p, q)-h f_{x}\left(p+h t_{0}, q+k t_{0}\right)-k f_{y}\left(p+h t_{0}, q+k t_{0}\right) \\
& =0
\end{aligned}
$$

which implies that $\left(\eta_{0}, \xi_{0}\right):=\left(p+h t_{0}, q+k t_{0}\right)$ is the unique two-dimensional Lagrange's mean value point of $f$ in $L$.

Furthermore, in view of (1), we get

$$
G_{f}^{\prime \prime}\left(t_{0}\right)=-F^{\prime \prime}\left(t_{0}\right)=-h^{2} f_{x x}\left(\eta_{0}, \xi_{0}\right)-2 h k f_{x y}\left(\eta_{0}, \xi_{0}\right)-k^{2} f_{y y}\left(\eta_{0}, \xi_{0}\right) \neq 0
$$

and $G_{f}^{\prime \prime}(t)$ is continuous. Hence, there exists a neighborhood $\left(t_{0}-r, t_{0}+r\right) \subset(0,1)$ of $t_{0}$ such that either $G_{f}^{\prime \prime}(t)>0$ for all $t \in\left(t_{0}-r, t_{0}+r\right)$ or $G_{f}^{\prime \prime}(t)<0$ for all $t \in\left(t_{0}-r, t_{0}+r\right)$. Since $G_{f}^{\prime}\left(t_{0}\right)=0$, $G_{f}^{\prime}(t)$ changes sign at $t_{0}$.

We now translate Theorem 2 into the statement (2) below by substituting as we see in the following table. (The function $H$ will be chosen later.)

\begin{tabular}{||c|c|c|c|c|c|c|c|c|c||}
\hline Theorem 2 & $f$ & $n$ & $t$ & $t_{0}$ & $t_{1}$ & $g$ & $N$ & $\varepsilon$ & $\delta$ \\
\hline \hline (2) below & $G_{f}$ & 1 & $t$ & $t_{0}$ & $t_{1}$ & $H$ & $\left(t_{0}-r, t_{0}+r\right)$ & $\tilde{\varepsilon}$ & $\tilde{\delta}$ \\
\hline
\end{tabular}

Regarding the table above, Theorem 2 states that

$$
\begin{aligned}
& \text { for any } \tilde{\varepsilon}>0 \text {, there corresponds a } \tilde{\delta}>0 \text { such that for any function } \\
& H: \mathbb{R} \rightarrow \mathbb{R} \text { differentiable in }\left(t_{0}-r, t_{0}+r\right) \text { with }\left|G_{f}(t)-H(t)\right|<\tilde{\delta} \\
& \text { for all } t \in\left(t_{0}-r, t_{0}+r\right) \text {, there exists a point } t_{1} \in\left(t_{0}-r, t_{0}+r\right) \\
& \text { satisfying } H^{\prime}\left(t_{1}\right)=0 \text { and }\left|t_{1}-t_{0}\right|<\tilde{\varepsilon} \text {. }
\end{aligned}
$$

For any given $\varepsilon>0$, let

$$
\tilde{\varepsilon}:=\frac{\varepsilon}{\sqrt{h^{2}+k^{2}}} \text { and } \delta:=\frac{\tilde{\delta}}{3}
$$

where $\tilde{\delta}>0$ is chosen such that the statement in (2) holds true. Let $g: \mathbb{R}^{2} \rightarrow \mathbb{R}$ be a partial differentiable function satisfying $|f(x, y)-g(x, y)|<\delta$ for all $(x, y) \in L$. If we define a differentiable function $G_{g}: \mathbb{R} \rightarrow \mathbb{R}$ by

$$
G_{g}(t):=\operatorname{tg}(u, v)+(1-t) g(p, q)-g(p+h t, q+k t)
$$


for all $t \in \mathbb{R}$, then it holds that

$$
\begin{aligned}
\left|G_{f}(t)-G_{g}(t)\right| \leq & |f(u, v)-g(u, v)|+|f(p, q)-g(p, q)| \\
& +|f(p+h t, q+k t)-g(p+h t, q+k t)| \\
< & 3 \delta \\
= & \tilde{\delta}
\end{aligned}
$$

for all $t \in\left(t_{0}-r, t_{0}+r\right) \subset(0,1)$.

Hence, by the statement in (2) with $H=G_{g}$, there exists a point $t_{1} \in\left(t_{0}-r, t_{0}+r\right)$ such that $G_{g}^{\prime}\left(t_{1}\right)=0$ and $\left|t_{1}-t_{0}\right|<\tilde{\varepsilon}$. We note that $G_{g}^{\prime}\left(t_{1}\right)=0$ implies that

$$
\begin{aligned}
G_{g}^{\prime}\left(t_{1}\right) & =g(u, v)-g(p, q)-h g_{x}\left(p+h t_{1}, q+k t_{1}\right)-k g_{y}\left(p+h t_{1}, q+k t_{1}\right) \\
& =g(u, v)-g(p, q)-(u-p) g_{x}\left(\eta_{1}, \xi_{1}\right)-(v-q) g_{y}\left(\eta_{1}, \xi_{1}\right) \\
& =0
\end{aligned}
$$

where $\left(\eta_{1}, \xi_{1}\right):=\left(p+h t_{1}, q+k t_{1}\right)$ is the point on the line segment $L$. Indeed, $\left(\eta_{1}, \xi_{1}\right)$ is a two-dimensional Lagrange's mean value point of $g$ in L. Moreover, it holds that

$$
\begin{aligned}
\left|\left(\eta_{0}, \xi_{0}\right)-\left(\eta_{1}, \xi_{1}\right)\right| & =\left|\left(p+h t_{0}, q+k t_{0}\right)-\left(p+h t_{1}, q+k t_{1}\right)\right| \\
& =\left|\left(h\left(t_{0}-t_{1}\right), k\left(t_{0}-t_{1}\right)\right)\right| \\
& =\sqrt{h^{2}+k^{2}}\left|t_{0}-t_{1}\right| \\
& <\sqrt{h^{2}+k^{2}} \tilde{\varepsilon} \\
& =\varepsilon .
\end{aligned}
$$

Hence, the point $\left(\eta_{1}, \xi_{1}\right)$ is a two-dimensional Lagrange's mean value point of $g$ in $L$ with $\left|\left(\eta_{0}, \xi_{0}\right)-\left(\eta_{1}, \xi_{1}\right)\right|<\varepsilon$, which completes the proof.

We are now interested in choosing an appropriate $\delta$ in Theorem 6 because the magnitude of $\delta$ seems to be important for the practical use of this theorem. We only need to apply the algorithm in Remark 1 and refer to the statement in (2) for the following algorithm.

Remark 2. For the notations $r, t_{0}, N=\left(t_{0}-r, t_{0}+r\right)$, and $G_{f}$, we refer the proof of Theorem 6 and we introduce an efficient algorithm for choosing the $\delta$ :

(i) by considering (3), we choose $\tilde{\varepsilon}$ such that $0<\tilde{\varepsilon}<r$;

(ii) we choose $t_{2}, t_{3}$ and $\alpha$ such that $\left|t_{2}-t_{0}\right|<\frac{\tilde{\varepsilon}}{2},\left|t_{3}-t_{0}\right|<\frac{\tilde{\varepsilon}}{2}, 0<\alpha<\frac{\tilde{\varepsilon}}{2}$, and $\Delta_{\alpha} G_{f}\left(t_{2}\right) \Delta_{\alpha} G_{f}\left(t_{3}\right)<0$;

(iii) we choose the $\tilde{\delta}$ as large as possible with $0<\tilde{\delta}<\min \left\{\frac{1}{2}\left|\Delta_{\alpha} G_{f}\left(t_{2}\right)\right|, \frac{1}{2}\left|\Delta_{\alpha} G_{f}\left(t_{3}\right)\right|\right\}$;

(iv) we determine $\delta$ by $\delta:=\frac{1}{3} \tilde{\delta}$.

The following two corollaries show that our main result (Theorem 6) is a generalization and an improvement of [16] (Theorem 2.2). If we put $v=q$ in Theorem 6, then the condition (1) is reduced to $f_{x x}\left(\eta_{0}, \xi_{0}\right) \neq 0$. Hence, we get the following corollary.

Corollary 1. Assume that $L$ is the line segment joining two distinct points $(p, q)$ and $(u, q)$ and that $f$ : $\mathbb{R}^{2} \rightarrow \mathbb{R}$ is a twice continuously partial differentiable function. Suppose $\left(\eta_{0}, \xi_{0}\right)$ is the unique two-dimensional Lagrange's mean value point of $f$ in $L$ and $f_{x x}\left(\eta_{0}, \xi_{0}\right) \neq 0$. Then, for any given $\varepsilon>0$, there exists a $\delta>0$ with the property that if a partial differentiable function $g: \mathbb{R}^{2} \rightarrow \mathbb{R}$ satisfies $|f(x, y)-g(x, y)|<\delta$ for all $(x, y) \in L$, then there is a two-dimensional Lagrange's mean value point $\left(\eta_{1}, \xi_{1}\right)$ of $g$ in $L$ with $\left|\left(\eta_{0}, \xi_{0}\right)-\left(\eta_{1}, \xi_{1}\right)\right|<\varepsilon$. In particular, $y=\xi=\xi_{0}=\xi_{1}=q$ throughout the statement of this corollary. 
If we put $u=p$ in Theorem 6 , then the condition (1) is reduced to $f_{y y}\left(\eta_{0}, \xi_{0}\right) \neq 0$. Thus, we obtain the following corollary.

Corollary 2. Assume that $L$ is the line segment joining two distinct points $(p, q)$ and $(p, v)$ and that $f$ : $\mathbb{R}^{2} \rightarrow \mathbb{R}$ is a twice continuously partial differentiable function. Suppose $\left(\eta_{0}, \xi_{0}\right)$ is the unique two-dimensional Lagrange's mean value point of $f$ in $L$ and $f_{y y}\left(\eta_{0}, \xi_{0}\right) \neq 0$. Then, for any given $\varepsilon>0$, there exists a $\delta>0$ with the property that if a partial differentiable function $g: \mathbb{R}^{2} \rightarrow \mathbb{R}$ satisfies $|f(x, y)-g(x, y)|<\delta$ for all $(x, y) \in L$, then there is a two-dimensional Lagrange's mean value point $\left(\eta_{1}, \xi_{1}\right)$ of $g$ in $L$ with $\left|\left(\eta_{0}, \xi_{0}\right)-\left(\eta_{1}, \xi_{1}\right)\right|<\varepsilon$. In particular, $x=\eta=\eta_{0}=\eta_{1}=p$ throughout the statement of this corollary.

\section{Example}

Assume that $L$ is the set of all points on the line segment joining the points $(-1,-1)$ and $(1,1)$ and that $f: \mathbb{R}^{2} \rightarrow \mathbb{R}$ is a twice continuously partial differentiable function defined by

$$
f(x, y):=x^{2}+y^{2} .
$$

Then we have $p=q=-1, u=v=1, h=u-p=2$, and $k=v-q=2$. In addition, $\left(\eta_{0}, \xi_{0}\right)=(0,0)$ is the unique two-dimensional Lagrange's mean value point of $f$ in $L$ and

$$
h^{2} f_{x x}\left(\eta_{0}, \xi_{0}\right)+2 h k f_{x y}\left(\eta_{0}, \xi_{0}\right)+k^{2} f_{y y}\left(\eta_{0}, \xi_{0}\right)=16 \neq 0,
$$

i.e., $f$ satisfies the condition (1).

Consulting the proof of Theorem 6, we now define $G_{f}(t)=t f(u, v)+(1-t) f(p, q)-f(p+$ $h t, q+k t)=8 t-8 t^{2}$. It then follows from the equation $G_{f}^{\prime}\left(t_{0}\right)=8-16 t_{0}=0$ that $t_{0}=\frac{1}{2}$. Moreover, $G_{f}^{\prime \prime}\left(t_{0}\right)=-16 \neq 0$. Thus, we conclude that $G_{f}^{\prime}(t)>0$ for all $t<t_{0}$ and $G_{f}^{\prime}(t)<0$ for all $t>t_{0}$, i.e., $G_{f}^{\prime}(t)$ changes sign at $t_{0}=\frac{1}{2}$ and we can choose an arbitrary $0<\tilde{\varepsilon}<\frac{1}{2}$ (we see $\left(t_{0}-r, t_{0}+r\right) \subset(0,1)$ in the proof of Theorem 6 and we take Remark 2 (i) into account).

We now follow Remark 2 (ii) and choose $t_{2}, t_{3}$, and $\alpha$ with $\left|t_{2}-t_{0}\right|=\left|t_{2}-\frac{1}{2}\right|<\frac{\tilde{\varepsilon}}{2},\left|t_{3}-t_{0}\right|=$ $\left|t_{3}-\frac{1}{2}\right|<\frac{\tilde{\varepsilon}}{2}$, and $0<\alpha<\frac{\tilde{\varepsilon}}{2}$ such that

$$
\Delta_{\alpha} G_{f}\left(t_{2}\right) \Delta_{\alpha} G_{f}\left(t_{3}\right)=8 \alpha\left(1-\alpha-2 t_{2}\right) 8 \alpha\left(1-\alpha-2 t_{3}\right)<0 .
$$

If we set $t_{2}=\frac{1}{2}-\frac{\tilde{\varepsilon}}{4}, t_{3}=\frac{1}{2}+\frac{\tilde{\varepsilon}}{4}$, and $\alpha=\frac{\tilde{\varepsilon}}{4}$, then we get

$$
\Delta_{\alpha} G_{f}\left(t_{2}\right) \Delta_{\alpha} G_{f}\left(t_{3}\right)=2 \tilde{\varepsilon} \times \frac{\tilde{\varepsilon}}{4} \times 2 \tilde{\varepsilon} \times\left(-\frac{3}{4} \tilde{\varepsilon}\right)<0 .
$$

Hence, by considering (3) and Remark 2 (iii), we can choose $\tilde{\delta}=\frac{\varepsilon^{2}}{33}$ which is consistent with

$$
0<\tilde{\delta}<\min \left\{\frac{1}{2}\left|\Delta_{\alpha} G_{f}\left(t_{2}\right)\right|, \frac{1}{2}\left|\Delta_{\alpha} G_{f}\left(t_{3}\right)\right|\right\}=\frac{\tilde{\varepsilon}^{2}}{4}=\frac{1}{4} \frac{\varepsilon^{2}}{h^{2}+k^{2}}=\frac{\varepsilon^{2}}{32} .
$$

According to Theorem 6 and Remark 2 (iv), for any given $\varepsilon>0$, if a partial differentiable function $g: \mathbb{R}^{2} \rightarrow \mathbb{R}$ satisfies $|f(x, y)-g(x, y)|<\frac{\varepsilon^{2}}{99}$ for all $(x, y) \in L$, then there exists a two-dimensional Lagrange's mean value point $\left(\eta_{1}, \xi_{1}\right)$ of $g$ in $L$ with $\left|\left(\eta_{0}, \xi_{0}\right)-\left(\eta_{1}, \xi_{1}\right)\right|<\varepsilon$.

\section{Discussions}

In this paper, we prove the Hyers-Ulam stability of two-dimensional Lagrange's mean value point:

Assume that $L$ denote the line segment joining two points in the plane and that $f: \mathbb{R}^{2} \rightarrow \mathbb{R}$ is a twice continuously partial differentiable function. Moreover, suppose $\left(\eta_{0}, \xi_{0}\right)$ is the unique two-dimensional Lagrange's mean value point of $f$ in $L$ and the condition (1) is fulfilled. Then, for any given $\varepsilon>0$, 
there exists a $\delta>0$ with the property that if a partial differentiable function $g: \mathbb{R}^{2} \rightarrow \mathbb{R}$ satisfies $|f(x, y)-g(x, y)|<\delta$ for all $(x, y) \in L$, then there exists a two-dimensional Lagrange's mean value point $\left(\eta_{1}, \xi_{1}\right)$ of $g$ in L with $\left|\left(\eta_{0}, \xi_{0}\right)-\left(\eta_{1}, \xi_{1}\right)\right|<\varepsilon$.

The main theorem of this paper is an extension and an improvement of a previous work [16] (Theorem 2.2). Indeed, even Corollary 1 or 2 is a generalization and an improvement of [16] (Theorem 2.2).

Moreover, we introduce an algorithm for determining an appropriate constant $\delta$ depending on $f$ and $\varepsilon$ only. The larger $\delta$ is chosen, the more efficiently we apply our main theorem in practical use. This method helps us to choose a 'large' $\delta$ such that if a partial differentiable function $g$ satisfies inequality $|f(x, y)-g(x, y)|<\delta$ for all $(x, y) \in L$, then there exists a two-dimensional Lagrange's mean value point $\left(\eta_{1}, \xi_{1}\right)$ of $g$ with $\left|\left(\eta_{0}, \xi_{0}\right)-\left(\eta_{1}, \xi_{1}\right)\right|<\varepsilon$.

Author Contributions: Writing—original draft, J.S.M. and K.J.H.; Writing—review \& editing, J.S.M. and K.J.H.

Funding: This research was supported by Basic Science Research Program through the National Research Foundation of Korea (NRF) funded by the Ministry of Education (No. 2016R1D1A1B03931061).

Conflicts of Interest: The authors declare no conflict of interest.

\section{References}

1. Ulam, S.M. A Collection of Mathematical Problems; Interscience Publishers: New York, NY, USA, 1960.

2. Hyers, D.H. On the stability of the linear functional equation. Proc. Natl. Acad. Sci. USA 1941, $27,222-224$. [CrossRef] [PubMed]

3. Brzdęk, J.; Popa, D.; Raşa, I. Hyers-Ulam stability with respect to gauges. J. Math. Anal. Appl. 2017, 453, 620-628. [CrossRef]

4. Brzdęk, J.; Popa, D.; Xu, B. The Hyers-Ulam stability of nonlinear recurrences. J. Math. Anal. Appl. 2007, 335, 443-449. [CrossRef]

5. Czerwik, S. Functional Equations and Inequalities in Several Variables; World Scientific Publishing: Singapore, 2002.

6. Forti, G.L. Hyers-Ulam stability of functional equations in several variables. Aequ. Math. 1995, 50, 143-190. [CrossRef]

7. Găvrutǎ, P. A generalization of the Hyers-Ulam-Rassias stability of approximately additive mappings. J. Math. Anal. Appl 1994, 184, 431-436. [CrossRef]

8. Hyers, D.H.; Isac, G.; Rassias, T.M. Stability of Functional Equations in Several Variables; Birkäuser: Boston, MA, USA, 1998.

9. Hyers, D.H.; Rassias, T.M. Approximate homomorphisms. Aequ. Math. 1992, 44, 125-153. [CrossRef]

10. Jung, S.-M. Hyers-Ulam-Rassias Stability of Functional Equations in Nonlinear Analysis; Springer: New York, NY, USA, 2011.

11. Jung, S.-M. Hyers-Ulam stability of a system of first order linear differential equations with constant coefficients. J. Math. Anal. Appl. 2006, 320, 549-561. [CrossRef]

12. Jung, S.-M.; Sahoo, P.K. On the stability of a mean value type functional equation. Demonstr. Math. 2000, 33, 793-796. [CrossRef]

13. Popa, D.; Raşa, I. On the Hyers-Ulam stability of the linear differential equation. J. Math. Anal. Appl. 2011, 381, 530-537. [CrossRef]

14. Qarawani, M.N. Hyers-Ulam stability for Mackey-Glass and Lasota differential equations. J. Math. Res. 2013, 5, 34-40. [CrossRef]

15. Rassias, T.M. On the stability of the linear mapping in Banach spaces. Proc. Am. Math. Soc. 1978, 72, $297-300$. [CrossRef]

16. Găvrută, P.; Jung, S.-M.; Li, Y. Hyers-Ulam stability of mean value points. Ann. Funct. Appl. 2010, 1 , 68-74. [CrossRef]

17. Ulam, S.M.; Hyers, D.H. On the stability of differential expressions. Math. Mag. 1954, 28, 59-64. [CrossRef]

18. Flett, T.M. A mean value theorem. Math. Gaz. 1958, 42 , 38-39. [CrossRef] 
19. Das, M.; Riedel, T.; Sahoo, P.K. Hyers-Ulam stability of Flett's points. Appl. Math. Lett. 2003, 16, $269-271$. [CrossRef]

20. Lee, W.; Xu, S.; Ye, F. Hyers-Ulam stability of Sahoo-Riedel's point. Appl. Math. Lett. 2009, 22, 1649-1652. [CrossRef]

21. Sahoo, P.K.; Riedel, T. Mean Value Theorems and Functional Equations; World Scientific: Singapore, 1998.

22. Courant, R. Differential and Integral Calculus, Volume II; Interscience Publishers: New York, NY, USA, 1964.

(C) 2018 by the authors. Licensee MDPI, Basel, Switzerland. This article is an open access article distributed under the terms and conditions of the Creative Commons Attribution (CC BY) license (http://creativecommons.org/licenses/by/4.0/). 